\title{
Medium to Long-Term Outcome of Thoracoscapular Arthrodesis with Screw Fixation for Facioscapulohumeral Muscular Dystrophy
}

\author{
Alexander Van Tongel, MD, Ehud Atoun, MD, Ali Narvani, FRCS, Giuseppe Sforza, MD, \\ Stephen Copeland, FRCS, and Ofer Levy, MD, MCh(Orth), FRCS \\ Investigation performed at the Reading Shoulder Unit, Royal Berkshire Hospital \\ and Berkshire Independent Hospital, Reading, Berkshire, United Kingdom
}

\begin{abstract}
Background: Shoulder girdle muscle weakness is the most constant feature of facioscapulohumeral muscular dystrophy and leads to scapular winging. Mechanical fixation of the scapula to the thoracic wall provides a stable fulcrum on which the deltoid muscle can exert its action on the humerus. The aim of this study was to evaluate the medium to longterm outcome of thoracoscapular arthrodesis with screw fixation (the modified Howard-Copeland technique).
\end{abstract}

Methods: All patients with facioscapulohumeral dystrophy who underwent thoracoscapular arthrodesis with screw fixation and bone-grafting from July 1997 to July 2010 were retrospectively reviewed. Preoperative and postoperative clinical assessment included active shoulder elevation, the Constant score, a patient satisfaction score, and cosmetic satisfaction. Union was determined both clinically and radiographically.

Results: Thoracoscapular arthrodesis was performed in thirty-five shoulders in twenty-four patients; eleven patients underwent bilateral procedures. The principal study group consisted of thirty-two shoulders in twenty-one patients with a minimum follow-up of twenty-four months (mean, eighty-eight months; range, twenty-four to 174 months). The mean Constant score increased from 30 (range, 17 to 41 ) preoperatively to 61 (range, 30 to 90) postoperatively. The mean satisfaction score increased from 1 (range, 0 to 4 ) to 8.4 (range, 4 to 10). Early complications consisted of one pneumothorax, one superficial wound infection, and four early failures, two of which were associated with noncompliance with the postoperative regimen. Late complications consisted of one posttraumatic fracture resulting in loosening and one painful nonunion; both were treated successfully with revision.

Conclusions: Thoracoscapular arthrodesis with screw fixation prevented scapular winging and improved short-term and long-term shoulder function in patients with facioscapulohumeral dystrophy.

Level of Evidence: Therapeutic Level IV. See Instructions for Authors for a complete description of levels of evidence.

$\mathrm{F}$ acioscapulohumeral muscular dystrophy is one of the most common types of muscular dystrophy, affecting seven in 1,000,000 individuals. It was first described by Landouzy and Dejerine in $1884^{1}$ and is a genetic disorder characterized by weakness in facial and shoulder girdle musculature ${ }^{2}$. It is an autosomal dominant myopathy, although $10 \%$ to $30 \%$ of cases arise from a de novo mutation ${ }^{3,4}$. Symptoms usually begin in childhood, and $>90 \%$ of patients have some evidence of disease

on examination by the age of twenty years. The course of the disease is slowly progressive, although many patients have long periods of relatively stable function ${ }^{3}$. Life expectancy is normal, but up to $15 \%$ of affected individuals become severely disabled and must eventually use a wheelchair ${ }^{1,3,5}$. Facioscapulohumeral dystrophy is currently untreatable.

Shoulder girdle weakness is the most constant feature of this disease and leads to scapular winging. Patients with

Disclosure: None of the authors received payments or services, either directly or indirectly (i.e., via his or her institution), from a third party in support of any aspect of this work. One or more of the authors, or his or her institution, has had a financial relationship, in the thirty-six months prior to submission of this work, with an entity in the biomedical arena that could be perceived to influence or have the potential to influence what is written in this work. No author has had any other relationships, or has engaged in any other activities, that could be perceived to influence or have the potential to influence what is written in this work. The complete Disclosures of Potential Conflicts of Interest submitted by authors are always provided with the online version of the article. 
The Journal of Bone \& Joint Surgery - jbjs.org Volume 95-A • Number $15 \cdot$ August 7, 2013
Medium to Long-Term Outcome of Thoracoscapular

Arthrodesis for Muscular Dystrophy facioscapulohumeral dystrophy usually have selective weakness of the thoracoscapular muscles with relative preservation of deltoid muscle function. When shoulder elevation is attempted using the deltoid, the scapula rotates and lifts off the chest wall, resulting in a loss of strength to move the glenohumeral joint and an inability to sustain shoulder abduction or flexion ${ }^{5}$. The patient is unable to hold heavy objects or perform activities that require arm elevation, such as combing hair or shaving. Gross winging of the scapula can be a substantial cosmetic deformity. Winging may pose problems for routine garment wear; shoulder straps on clothes tend to slip off the shoulder. Winging of the scapula may also cause pain in the shoulder girdle ${ }^{5}$.

Mechanical fixation of the scapula to the thoracic wall provides a stable fulcrum on which the deltoid muscle can exert its action on the humerus, allowing abduction of the arm at the shoulder joint without rotation of the scapula ${ }^{1}$. Various techniques to achieve fixation have been published. We report the medium to long-term outcome of thoracoscapular arthrodesis with screw fixation (the modified Howard-Copeland technique).

\section{Materials and Methods}

We retrospectively reviewed all patients with facioscapulohumeral dystrophy Who had undergone thoracoscapular arthrodesis with screw fixation and bone-grafting from July 1997 to July 2010 at our institution. The diagnosis was established on the basis of clinical evaluations, electromyographic findings, and genetic tests when available.

We had recommended thoracoscapular arthrodesis for patients who had difficulties with activities that required arm elevation and had active abduction and active forward flexion of $30^{\circ}$ to $90^{\circ}$ that increased to $\geq 100^{\circ}$ when the scapula was held manually in place on the thorax (the Horwitz maneuver) ${ }^{6,7}$. Patients who could not lift the arm when the scapula was held manually on the thorax (muscle strength $2 / 5$ or less according to the Medical Research Council $[\mathrm{MRC}]$ grading system) were considered to have a contraindication for thoracoscapular arthrodesis. All patients in the series had deltoid muscle strength of at least $3 / 5$ to $4 / 5$ preoperatively. The two senior surgeons (O.L., S.C.) performed all surgical procedures.

\section{Surgical Technique}

The patient is positioned prone at the edge of the operating table under general anesthesia. The arms are positioned in $90^{\circ}$ of elevation in the scapular plane and $90^{\circ}$ of external rotation on a board (see Appendix). Fixation of the scapula in this position will allow the best range of postoperative shoulder movement. The upper extremity is draped free to permit easy intraoperative handling. The ipsilateral posterior iliac crest is also draped free for graft harvesting. A longitudinal incision, parallel to the spinous processes of the thoracic vertebrae, is made over the medial border of the scapula. The trapezius muscle is incised parallel to the skin incision. The levator scapula, rhomboid major, and rhomboid minor muscles are detached from the medial border of the scapula and retracted medially. The supraspinatus, infraspinatus, and teres minor muscles are detached subperiosteally from the medial border of the scapula to expose a 3-cm-wide strip on the medial side of the scapula (see Appendix). The subscapularis muscle is detached subperiosteally from the medial 5 to $6 \mathrm{~cm}$ of the scapula, and the impinging part of the muscle is resected to allow better contact between the scapula and the ribs.

Three of four ribs lying under the most convenient part of the scapula are chosen; these are usually the fourth, fifth, and sixth ribs (the third or the seventh may be fixed accordingly if needed). The first chosen rib (usually the fourth rib) is the rib nearest to the scapular spine, as this is the location of the best and strongest bone on the medial aspect of the scapula (see Appendix).

Preparation of each rib is begun by incising the periosteum in the line of the rib and separating the periosteum and parietal pleura on the deep surface of the rib (with the subperiosteal access performed from the superior part of the rib to avoid injury to the neurovascular bundle at the inferior aspect of the rib) so that a thin retractor (McDonald dissector) can be placed under the rib to prevent damage to the pleura. The superficial surface of the rib is petaled gently with a ferret gouge and partly decorticated. The scapula is placed in the correct position over the denuded rib. The scapula and underlying rib are drilled with a protective McDonald dissector in place to prevent damage to the pleura. The rib is then measured for appropriate screw length, and a $4.5-\mathrm{mm}$ self-tapping cortical or cancellous screw with a washer is inserted finger-tight. The second and third screws (and, if it is needed, the fourth screw) are inserted in a similar manner. It is best to mark the point to be drilled on the second rib with the first screw in place, then unscrew the first screw and complete the drilling of the rib with a protective McDonald dissector in place to prevent damage to the pleura. Likewise, the third hole is drilled with the previous two screws in place so that the scapula is fixed during the drilling; this process results in the best placement of the screws in the ribs (see Appendix). We use 4.5-mm cortical screws with washers. If the screw grip in the rib is not optimal, we use a cancellous screw. All of the screws have bicortical fixation in the ribs.

Bone graft is obtained by harvesting cortical and cancellous bone from the ipsilateral posterior iliac crest, or tricalcium phosphate granules are used as a bone graft substitute (see Appendix). The screws in the ribs are loosened, and bone chips (or bone graft substitute) are packed between the ribs and the deep surface of the scapula (see Appendix). The screws are tightened until stable fixation of the scapula to the ribs is achieved, and the wound is closed.

The shoulder is immobilized in an adjustable brace with the arm in $60^{\circ}$ of abduction and $30^{\circ}$ of forward flexion with the hand in front of the mouth. The arm is held in this position with the adjustable shoulder brace for three months. During this time, the patient is encouraged to perform elbow and hand movements within the brace. The brace is removed at three months after surgery. The patient is advised to use a pillow under the arm for about a week to bring the arm down gradually from the abducted position. Physical therapy begins at three months with passive motion followed by active exercises in elevation and rotation. Physiotherapy continues with exercises to restore glenohumeral movement and strengthen the deltoid muscle.

\section{Assessment}

Patient demographic information was collected and complications were recorded. Preoperative and postoperative clinical assessments included active shoulder elevation, the Constant score ${ }^{8}$, the Subjective Shoulder Value (SSV) ${ }^{9}$ (a patient satisfaction measure), and questions regarding cosmetic satisfaction. Union was determined both clinically and with use of anteroposterior and trans-scapular radiographs (Fig. 1).

\section{Source of Funding}

No external funding was received for the study.

\section{Results}

Thirty-five shoulders in twenty-four patients with facioscapulohumeral dystrophy were treated with thoracoscapular arthrodesis from July 1997 to July 2010 (see Appendix). In the eleven patients who underwent bilateral procedures, the side with more severe involvement was treated first, or the dominant side was treated first if the patient had symmetric involvement. The mean interval between bilateral procedures was sixteen months (range, six to thirty-six months). The mean age of the twenty-four patients at the time of the surgery was twenty-six years (range, sixteen to sixty-two years).

Three patients were lost to follow-up less than six months after surgery and were excluded from the medium to long-term evaluation but were included in the evaluation of complications. Two of these three patients (patients 6 and 7) had unsatisfactory outcomes. The third patient (patient 14) had a good early result 
The Journal of Bone \& Joint Surgery - Jbjs. org Volume 95-A • Number $15 \cdot$ August 7, 2013

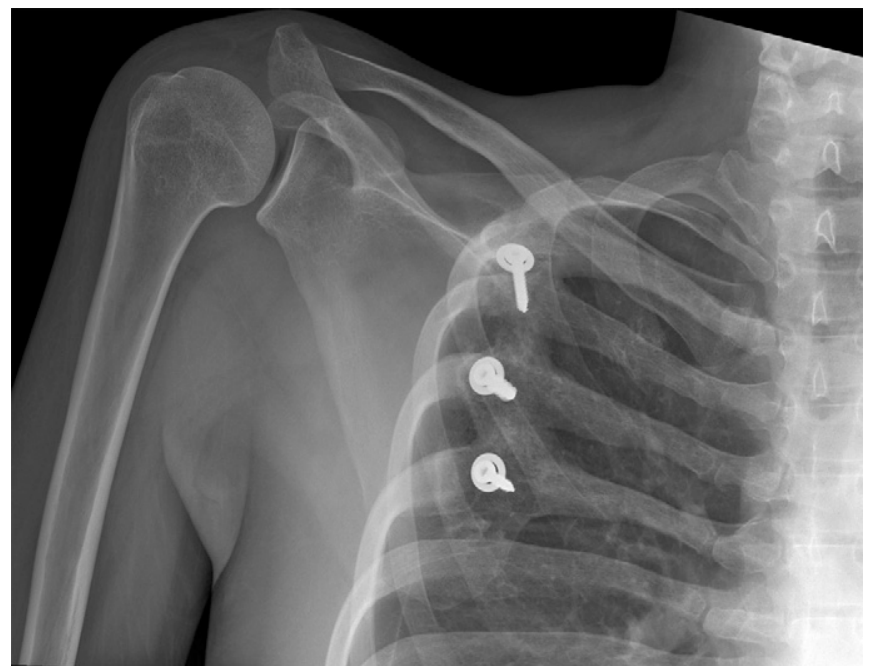

Fig. 1-A

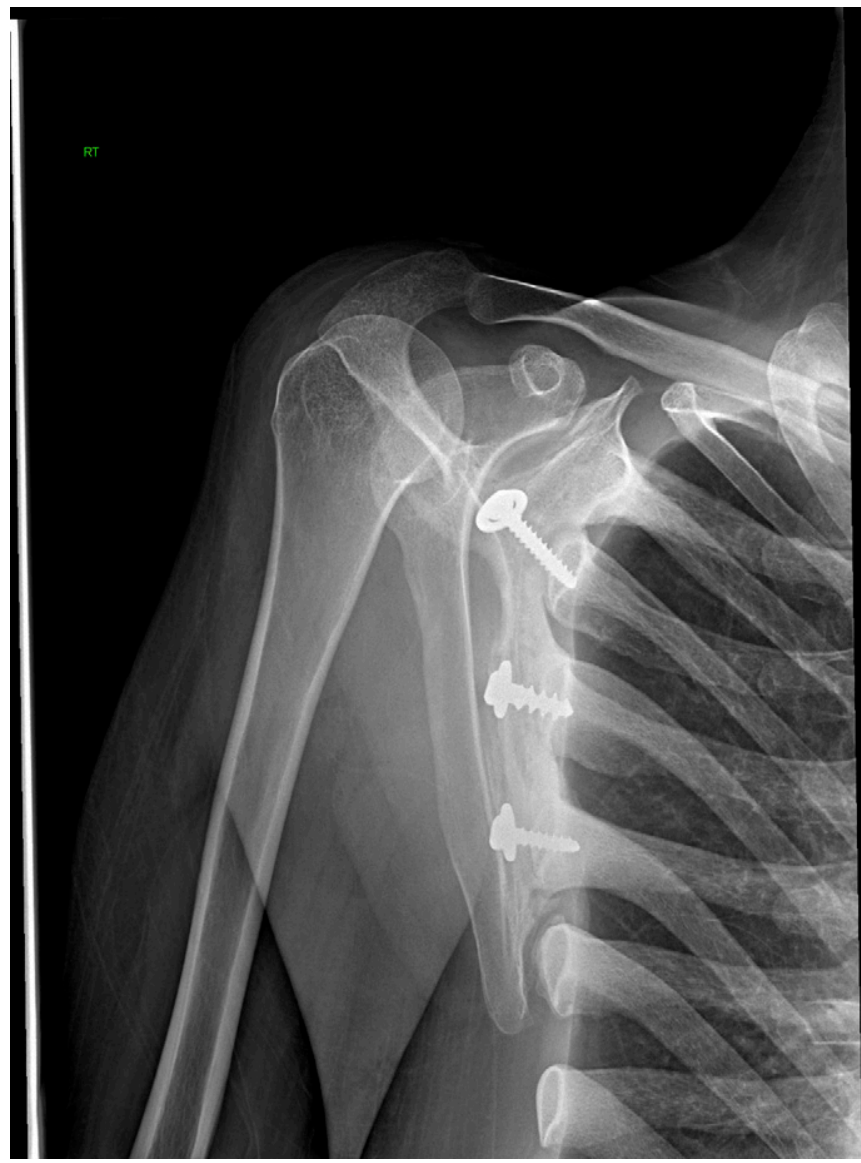

Fig. 1-B

Anteroposterior (Fig. 1-A) and trans-scapular (Fig. 1-B) radiographs showing union after thoracoscapular arthrodesis using screw fixation.

four months after surgery but emigrated and could not be followed further.

The remaining thirty-two shoulders in twenty-one patients (fourteen male and seven female) formed the principal study group. All of these patients had been followed for a
Medium to Long-Term Outcome of Thoracoscapular

Arthrodesis for Muscular Dystrophy minimum of twenty-four months (mean, eighty-eight months; range, twenty-four to 174 months) (see Appendix).

Mean active arm elevation increased from $65^{\circ}$ preoperatively to $119^{\circ}$ postoperatively. The mean Constant score increased from 30 points (range, 17 to 41 points) preoperatively to 61 points (range, 30 to 90 points) postoperatively. The mean pain subscore improved from 9.8 (range, 3 to 15 ) of 15 points preoperatively to 13.2 points (range, 8 to 15 points) postoperatively. The mean subscore for activities of daily living improved from 5.4 (range, 2 to 13 ) of 20 points preoperatively to 14.6 points (range, 6 to 20 points) postoperatively. The mean subscore for range of shoulder motion improved from 14.6 (range, 8 to 22) of 40 points preoperatively to 25.6 points (range, 12 to 36 points) postoperatively. The mean strength subscore improved from 0 of 25 points preoperatively to 8 points (range, 0 to 20 points) postoperatively. The mean SSV (patient satisfaction) score improved from 1 (range, 0 to 4 ) of 10 points preoperatively to 8.4 points (range, 4 to 10 points) postoperatively.

A comparison between the functional outcomes in the patients with the longest follow-up of more than ten years (eight shoulders) and in those with a shorter follow-up of two to five years (twelve shoulders) revealed no difference in outcome. The mean Constant score was 62.5 points (an improvement of 34.1 points from 28.4 points preoperatively) in the group with the longest follow-up (more than ten years) compared with 59 points (an improvement of 28 points from 31 points preoperatively) in the group with shorter follow-up.

\section{Cosmesis}

Before surgery, the scapulae were not only winged but elevated during attempted shoulder flexion or abduction, resulting in an unsightly appearance of widening of the base of the neck. Although fixation of the scapula leaves a considerable scar, the patients had a general improvement in appearance. The position in which the scapula was fixed in these patients maximizes the range of active movement. This position is farther lateral than the natural position of the scapula and tends to produce a slightly "squared shoulder appearance." The patients did not complain about this appearance, and the female patients liked it as it kept clothing straps and handbag shoulder straps from falling.

\section{Early Complications}

One patient (one shoulder) had a pneumothorax that resolved spontaneously. One patient (one shoulder) had a superficial wound infection that was treated successfully with oral antibiotics. Four patients (four shoulders) had signs of early failure. One patient (patient 3 ) had removed the brace in the first few days after surgery, resulting in early pullout of the screws; revision of the fixation resulted in successful fusion. Another patient (patient 7) also removed the brace in the early postoperative period, contrary to instructions, resulting in pullout of the screws and loss of fixation. He underwent revision approximately a week after the index procedure but again removed the brace after a few days and had a second failure of the fixation. He was not offered further treatment and was lost to follow-up. Two patients (patients 13 and 23) had signs of nonunion with early 
The Journal of Bone \& Joint Surgery $\cdot$ Jbjs.org Volume 95-A · Number $15 \cdot$ August 7, 2013
Medium to Long-Term Outcome of Thoracoscapular

Arthrodesis for Muscular Dystrophy loosening of the screws; revision resulted in a solid fusion. These four patients were all overweight (mean body mass index, $34 \mathrm{~kg} / \mathrm{m}^{2}$ ) and male.

\section{Late Complications}

One patient (one shoulder, patient 13) fell fifteen months after surgery and sustained a fracture of the scapula and pullout of the screws resulting in loosening. He underwent revision involving plate and screw fixation with a good result. One patient (one shoulder, patient 6) developed a painful nonunion and was revised successfully elsewhere. One patient (one shoulder, patient 16) died a year after surgery because of an unrelated acute cardiac event. At that time, she had been very pleased with the arthrodesis on the first side and was scheduled to undergo arthrodesis on the contralateral side. One patient (one shoulder, patient 22) had rapid deterioration of all upper and lower-limb muscle function resulting in severe weakness of unknown origin several months after surgery that had resulted in successful fusion but a poor outcome.

\section{Radiographic Assessment}

Radiographic evaluation of the principal study group (thirtytwo shoulders followed for at least two years) at the time of the latest follow-up showed clear callus formation without signs of screw loosening in all but two patients (Fig. 1). Two patients (patients 13 and 23) who had shown earlier signs of nonunion with loosening of the screws had undergone revision resulting in solid fusion at the time of the latest follow-up. Fusion with callus formation was observed equally with the use of autologous bone graft and bone graft substitute.

\section{Discussion}

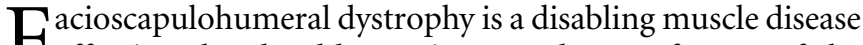

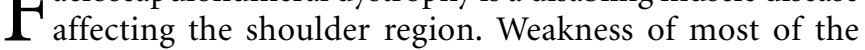
shoulder girdle muscles results in winging and instability of the scapula during attempted shoulder elevation and abduction. Mechanical fixation of the scapula to the ribs has been proposed as an effective solution for this problem. It provides the deltoid muscle with a stable anchor point on which to function, prevents scapular winging, and improves the function of the deltoid muscle in these patients.

Surgical intervention in patients with facioscapulohumeral dystrophy typically consists of either thoracoscapular arthrodesis (scapulodesis) ${ }^{1,10-20}$ or thoracoscapular soft-tissue fixation without arthrodesis (scapulopexy) ${ }^{4,21,22}$ (see Appendix). Arthrodesis of the scapula to the thoracic wall typically yields a solid fusion; however, there is concern regarding reduced respiratory function as well as the requirement for a prolonged period of postoperative immobilization that may result in muscle atrophy. Scapulopexy, with its use of less rigid fixation, is believed to avoid the problem of reduced respiratory function, and the shoulder can be mobilized almost immediately postoperatively. Unfortunately, the soft-tissue fixation tends to stretch and loosen with time, resulting in an outcome that is less satisfactory overall ${ }^{5}$.

The patients in the present study had substantial improvement in active shoulder elevation as well as in endurance when holding the arm elevated. This is in accordance with most previous series (see Appendix). The active elevation in the present series appears to be slightly better than that in some of the other series; we attribute this improved range of motion to the technique for positioning the scapula on the thorax in the present study.

The mean Constant score in the present study improved substantially to 61 points (range, 30 to 90 points) at the time of the latest follow-up. Comparing the outcome of the group with the longest follow-up (more than ten years) with that of the group with two to five years of follow-up revealed no obvious deterioration of function over time in these patients. Our results are comparable with those in a previous study by our group in 1999, in which the mean postoperative Constant score was 58 points (range, 13 to 90 points) ${ }^{1}$.

Several other authors have used different functional scoring systems for postoperative evaluation. Rhee and Ha used the UCLA (University of California Los Angeles) shoulder score, which improved from a mean of 18.4 points preoperatively to 27.9 points at the time of the latest follow-up ${ }^{20}$. Demirhan et al. used the DASH (Disabilities of the Arm, Shoulder and Hand) score, which improved from a mean of $33.6 \pm 8.9$ points to $11.6 \pm 8.0$ points postoperatively ${ }^{2}$.

Pain is not usually a major indication for recommending surgery in patients with facioscapulohumeral dystrophy. However, in contrast to other opinions ${ }^{4,21}$, the present study showed that this condition also has an important pain component. Relief of pain has been described before ${ }^{14,15}$, but to our knowledge the present study is the first to have quantified this improvement.

The patients in the present study had a clear improvement in activities of daily living, as most went from having severe limitation to moderate limitation or less during daily and leisure activities. This is in agreement with previous studies ${ }^{1,13-15}$. Once fusion has been achieved, we do not expect future problems resulting from the fusion; however, facioscapulohumeral dystrophy is a progressive disease and shoulder function may also deteriorate if involvement of the deltoid and/or trapezius muscles worsens.

The patients in the present study were very pleased with the functional and the cosmetic results of the operation. The resulting position of the scapula is farther lateral than the natural position of the scapula and tends to produce a slightly "squared shoulder" appearance, although the patients did not complain about this appearance. The mean overall satisfaction with the involved shoulder improved from 1 to 8.4 of 10 points.

The thoracoscapular region is prone to perioperative complications. Intraoperative and postoperative complications such as pneumothorax, hemothorax, rib fracture, and scapular fracture have been reported ${ }^{1,11,13-17,19}$. One patient (one shoulder) in the present series had a pneumothorax that was treated successfully by conservative means. The rate of lung injury and pneumothorax associated with the screw fixation technique was low (one of thirty-five shoulders) in the present series compared with the higher prevalences in other series involving wire fixation (see Appendix). Furthermore, use of screws rather than wires around the ribs requires only a small drill hole in the rib, resulting in a lower risk of rib fracture ${ }^{11,14,19}$. One patient had a superficial wound infection due to rubbing of the brace on the wound. 
The Journal of Bone \& Joint Surgery $\cdot$ JBjS. Org Volume 95-A • Number $15 \cdot$ August 7, 2013
Medium to Long-Term Outcome of Thoracoscapular

Arthrodesis for Muscular Dystrophy
The outcomes of thoracoscapular arthrodesis in the patients who obtained fusion were not better than those of the alternative techniques. However, the fusion rate in the present series was $86 \%$ (thirty of thirty-five), equal to the rate in our previous series ${ }^{1}$. This is higher than the range of $58 \%$ to $83 \%$ in other published series involving wire fixation (see Appendix) ) $^{2,11,14,19}$.

Four shoulders in the present series had failure of the fixation during the early postoperative period. We attribute two of these failures to noncompliance with postoperative brace wear and activity restriction. There was no clear cause for the early failure in the other two patients. Commonalities in these cases were noted, as all of the patients were men, two were in their thirties, and all were overweight.

Late complications included one nonunion (3\%) and one late fracture due to acute trauma, both requiring revision. The rate of revision for nonunion has been reported to be $9 \%$ to $11 \%$ in some previous series ${ }^{2,10}$. Demirhan et al. ${ }^{2}$ attributed most of the nonunions in their series to vigorous activity early during the recommended immobilization period. Our patient followed the postoperative regimen adequately and his nonunion had no clear cause.

Selecting the number of ribs involved in the arthrodesis is controversial $^{2,11}$. We fused three consecutive ribs, providing adequate stability of the initial fixation. We believe that there is no need for a four or five-level arthrodesis, as the flat scapula cannot be approximated to the convex thorax at both ends.

We did not measure the preoperative and postoperative respiratory function of the patients in the present study. Such measurements by Copeland and Howard ${ }^{10}$ revealed only minimal loss of vital capacity. This was confirmed by Bunch and Siegel ${ }^{13}$, who found that the forced vital capacity had dropped to $74 \%$ of the predicted value; they attributed the drop to the rigidity of the bilateral arthrodeses in their patient. Jakab and Gledhill ${ }^{23}$ reported a mean decrease in vital capacity of $0.213 \mathrm{~L}$ after unilateral arthrodesis and an additional decrease of $0.05 \mathrm{~L}$ after bilateral arthrodeses. There was no change in forced expiratory volume after unilateral arthrodesis, but a decrease of 0.12 L was seen after bilateral arthrodeses.

In conclusion, thoracoscapular arthrodesis using screw fixation (the modified Howard-Copeland technique) prevented scapular winging and improved both short-term and long-term shoulder function in patients with facioscapulohumeral dystrophy.

\section{Appendix}

eA Figures illustrating the surgical technique and its outeA come as well as tables summarizing patient demographics, patient outcomes, and published reports on scapular fixation for facioscapulohumeral dystrophy are available with the online version of this article as a data supplement at jbjs.org.

Alexander Van Tongel, MD

Ehud Atoun, MD

Ali Narvani, FRCS

Giuseppe Sforza, MD

Stephen Copeland, FRCS

Ofer Levy, MD, MCh(Orth), FRCS

Reading Shoulder Unit,

Royal Berkshire Hospital,

London Road, Reading,

Berkshire RG1 5AN, United Kingdom.

E-mail address for O. Levy: oferlevy@readingshoulderunit.com

\section{References}

1. Copeland SA, Levy O, Warner GC, Dodenhoff RM. The shoulder in patients with muscular dystrophy. Clin Orthop Relat Res. 1999 Nov;368:80-91.

2. Demirhan M, Uysal O, Atalar AC, Kilicoglu O, Serdaroglu P. Scapulothoracic arthrodesis in facioscapulohumeral dystrophy with multifilament cable. Clin Orthop Relat Res. 2009 Aug;467(8):2090-7. Epub 2009 Mar 31.

3. Kissel JT. Facioscapulohumeral dystrophy. Semin Neurol. 1999;19(1):35-43.

4. Giannini S, Faldini C, Pagkrati S, Grandi G, Digennaro V, Luciani D, Merlini L. Fixation of winged scapula in facioscapulohumeral muscular dystrophy. Clin Med Res. 2007 Oct;5(3):155-62.

5. Orrell RW, Copeland S, Rose MR. Scapular fixation in muscular dystrophy. Cochrane Database Syst Rev. 2010;1:CD003278. Epub 2010 Jan 20.

6. Bunch WH. Scapulo-thoracic fusion. Minn Med. 1973 May;56(5):391-4.

7. Horwitz MT, Tocanitus LM. Isolated paralysis of the serratus anterior (magnus) muscle. J Bone Joint Surg Am. 1938 Jul;20(3):720-5.

8. Constant CR, Murley AH. A clinical method of functional assessment of the shoulder. Clin Orthop Relat Res. 1987 Jan;214:160-4.

9. Gilbart MK, Gerber C. Comparison of the subjective shoulder value and the Constant score. J Shoulder Elbow Surg. 2007 Nov-Dec;16(6):717-21.

10. Copeland SA, Howard RC. Thoracoscapular fusion for facioscapulohumeral dystrophy. J Bone Joint Surg Br. 1978 Nov;60(4):547-51.

11. Letournel E, Fardeau M, Lytle JO, Serrault M, Gosselin RA. Scapulothoracic arthrodesis for patients who have fascioscapulohumeral muscular dystrophy. J Bone Joint Surg Am. 1990 Jan;72(1):78-84.

12. Kocialkowski A, Frostick SP, Wallace WA. One-stage bilateral thoracoscapular fusion using allografts. A case report. Clin Orthop Relat Res. 1991 Dec;273:264-7. 13. Bunch WH, Siegel IM. Scapulothoracic arthrodesis in facioscapulohumeral muscular dystrophy. Review of seventeen procedures with three to twenty-one-year follow-up. J Bone Joint Surg Am. 1993 Mar;75(3):372-6.
14. Twyman RS, Harper GD, Edgar MA. Thoracoscapular fusion in facioscapulohumeral dystrophy: clinical review of a new surgical method. J Shoulder Elbow Surg. 1996 May-Jun;5(3):201-5.

15. Andrews CT, Taylor TC, Patterson VH. Scapulothoracic arthrodesis for patients with facioscapulohumeral muscular dystrophy. Neuromuscul Disord. 1998 Dec;8(8):580-4.

16. Alshameeri ZA, Garg $S$, Wallace WA. Scapulothoracic fusion for fascio-scapulohumeral dystrophy. Shoulder \& Elbow. 2011 Jan;3(1):56-61.

17. Ziaee MA, Abolghasemian M, Majd ME. Scapulothoracic arthrodesis for winged scapula due to facioscapulohumeral dystrophy (a new technique). Am J Orthop (Belle Mead NJ). 2006 Jul;35(7):311-5.

18. Diab M, Darras BT, Shapiro F. Scapulothoracic fusion for facioscapulohumeral muscular dystrophy. J Bone Joint Surg Am. 2005 Oct;87(10):2267-75.

19. Berne D, Laude F, Laporte C, Fardeau M, Saillant G. Scapulothoracic arthrodesis in facioscapulohumeral muscular dystrophy. Clin Orthop Relat Res. 2003 Apr;409:106-13.

20. Rhee YG, Ha JH. Long-term results of scapulothoracic arthrodesis of facioscapulohumeral muscular dystrophy. J Shoulder Elbow Surg. 2006 Jul-Aug;15(4): 445-50.

21. Giannini S, Ceccarelli F, Faldini C, Pagkrati S, Merlini L. Scapulopexy of winged scapula secondary to facioscapulohumeral muscular dystrophy. Clin Orthop Relat Res. 2006 Aug;449:288-94.

22. Ketenjian AY. Scapulocostal stabilization for scapular winging in facioscapulohumeral muscular dystrophy. J Bone Joint Surg Am. 1978 Jun;60(4):476-80.

23. Jakab E, Gledhill RB. Simplified technique for scapulocostal fusion in facioscapulohumeral dystrophy. J Pediatr Orthop. 1993 Nov-Dec;13(6):749-51. 24. Howard RC. Thoraco-scapular arthrodesis. J Bone Joint Surg Br. 1961;43(1):175. 